\title{
Cost-Utility of "Doxorubicin and Cyclophosphamide" versus "Gemcitabine and Paclitaxel" for Treatment of Patients with Breast Cancer in Iran
}

\author{
Nahid Hatam ${ }^{1}$, Mehrdad Askarian ${ }^{2}$, Javad Javan-Noghabi $^{3}$, Niloofar Ahmadloo ${ }^{4 *}$, \\ Mohammad Mohammadianpanah ${ }^{5}$
}

\begin{abstract}
Purpose: A cost-utility analysis was performed to assess the cost-utility of neoadjuvant chemotherapy regimens containing doxorubicin and cyclophosphamide (AC) versus paclitaxel and gemcitabine (PG) for locally advanced breast cancer patients in Iran. Materials and Methods: This cross-sectional study in Namazi hospital in Shiraz, in the south of Iran covered 64 breast cancer patients. According to the random numbers, the patients were divided into two groups, 32 receiving $\mathrm{AC}$ and 32 PG. Costs were identified and measured from a community perspective. These items included medical and non-medical direct and indirect costs. In this study, a data collection form was used. To assess the utility of the two regimens, the European Organization for Research and Treatment of Cancer Quality of Life Questionnaire-Core30 (EORTC QLQ-C30) was applied. Using a decision tree, we calculated the expected costs and quality adjusted life years (QALYs) for both methods; also, the incremental cost-effectiveness ratio was assessed. Results: The results of the decision tree showed that in the AC arm, the expected cost was 39,170 US\$ and the expected QALY was 3.39 and in the PG arm, the expected cost was 43,336 dollars and the expected QALY was 2.64 . Sensitivity analysis showed the cost effectiveness of the $A C$ and ICER=-5535 US\$. Conclusions: Overall, the results showed that AC to be superior to PG in treatment of patients with breast cancer, being less costly and more effective.
\end{abstract}

Keywords: Breast cancer - cost-utility - neoadjuvant therapy - doxorubicin - cyclophosphamide - gemcitabine - paclitaxel

Asian Pac J Cancer Prev, 16 (18), 8265-8270

\section{Introduction}

Cancer is an important public health and economic concern around the world. According to the World Health Organization report, over 22 million people in the world live with cancer and Global cancer incidence in 2004 was 11.4 million and this figure increases every year. Cancer is a second leading cause of death in the United States. 1.7 million cancer deaths are estimated in Europe in only one year (Ferlay et al., 2007). In Iran, cancer is the third leading cause of death (Mousavi et al., 2009). Cancer treatment costs represent quite a burden for the National Health Systems' budget. For example, the United States spends $16 \%$ of its gross domestic product on health care costs and $5 \%$ of that goes to cancer treatment costs (Marsland et al., 2010). Of all the cancers, breast cancer (BC) is the most common cancer and the leading cause of cancer death in females worldwide, and billions of dollars are spent each year for its treatment (Jemal et al., 2011). Despite advances in the diagnosis of breast cancer, locally advanced breast cancer continues to be a major clinical problem, particularly in developing countries (Papadimitriou et al., 2010). The incidence of this disease increases 1 to $2 \%$ per year in developed countries and $5 \%$ per year in less developed countries. In 2011, there were about 2.6 million breast cancer survivors in the United States and 39520 deaths have been estimated due to breast cancer in women in the U.S (Kolahdoozan et al., 2010). Breast cancer was increasing over the years in Iran and is now ranked as the first among cancers diagnosed in women. Based on the latest statistics announced by the Health Ministry of Iran, 27 people per hundred thousand women are diagnosed with breast cancer (Mohammadianpanah et al., 2012).

Nevertheless, breast cancer is much more serious in Iran, because the age of breast cancer in Iranian women compared to women in Western countries is lower than a decade. Lower age of incidence is very important and causes loss of more QALY in the health care system (Armour et al., 1996; Mohammadianpanah et al., 2012). One of the most common treatments for cancer is chemotherapy that causes long-term improvement in these patients. Despite the systematic destruction of the cancer cell, it also affects healthy cells. The toxicity

${ }^{1}$ Department of Health Administration, School of Management and Information Sciences, ${ }^{2}$ Department of Community Medicine, Shiraz, Nephro-Urology Research Center, ${ }^{3}$ Department of Health Economics, School of Management and Information Sciences, ${ }^{4}$ Department of Radiation Oncology, Namazi Hospital, ${ }^{5}$ Colorectal Research Center, Shiraz University of Medical Sciences, Shiraz, Iran ${ }^{\circledR}$ Equal contributors*For correspondence: ahmadloon@gmail.com 
of chemotherapy drugs causes side effects such as bone marrow suppression and immune system, liver toxicity, skin disorders, central nervous system disorders, genitourinary and gastrointestinal complications such as inflammation of the lining of the mouth and intestines (Del Gaudio and Menonna-Quinn, 1998; Mohammadianpanah et al., 2012).

Nowadays, the use of neoadjuvant chemotherapy tends to be more, because it causes a reduction in the size of the primary tumor, removal of rooting small and tumor sensitivity to chemotherapy, and increases the chance of surgery (Mohammadianpanah et al., 2012). Standard chemotherapy regimens for advanced breast cancer patients is drug regimen containing doxorubicin group which, according to several studies, leads to a reduction in tumor recurrence and increased prognosis of patients . Newly taxane drugs as neoadjuvant regimens is used hoping to get a better response and in neoadjuvant conditions they have shown a good activity and acceptable toxicity and lead to increased pathological complete response (Younis et al., 2011; Mohammadianpanah et al., 2012).

Different chemotherapy regimens are used for patients with advanced breast cancer and the adoption of a chemotherapeutic regimen into oncologic practice is a function of both its clinical and economic impacts on cancer management (Shih and Halpern, 2008). In the United States, the average cost for treatment of breast cancer per patient is $35,568 \mathrm{US} \$$ and that of treatment per patient per month was estimated 2896 US\$ . Research suggests that in Africa and Asia breast cancer treatment costs in stages I, II, or III are less than 390 US\$ per QALY, whereas in stage IV breast cancer treatment costs is more than 3,500 US\$ per QALY (Groot et al., 2006).

In Iran, medical direct costs for treatment of breast cancer in stages III and IV are significantly higher than in other stages, so that the average costs in four-stage breast cancer are 4190.9,4308.2, 5388, 4422.2 US\$, respectively (Davari et al., 2013).

Cancer can cause various complications and has different effects on the quality of life, Also, studies show that different chemotherapy treatments with different doses have different effects on patients' utility (Hornberger and Best, 2005).

This documentation highlights the importance of the use of economic evaluation techniques to determine the most appropriate treatment strategy for these patients. Economic evaluation compares the costs and outcomes of health interventions. To achieve the best options and its overall objective, we need to maximize the benefits with respect to resource constraints. Therefore, according to various medical expenses for treatment of breast cancer, and consequently, different economic and financial burden on the health system, this study aimed to evaluate the costutility of chemotherapy regimens containing doxorubicin and cyclophosphamide compared to gemcitabine and paclitaxel.

\section{Materials and Methods}

This is an economic evaluation study done in a cross- sectional method on 64 women suffering from breast cancer in Nemazee hospital in Shiraz, in the south of Iran using the following equation in which $\mathrm{Z1}^{-\alpha} / 2=1.96, \mathrm{Z1}^{-\beta}=$ $0.85, \delta 1=20, \delta 2=20, \mu 1=75, \mu 2=65$.

$$
\begin{gathered}
n=\left[\left(Z_{1-}{ }_{2}^{+} Z_{1-\beta}\right)^{2}\left(\delta_{1}^{2}+\delta_{2}^{2}\right)\right] \\
\left(\mu_{1}-\mu_{2}\right)^{2}
\end{gathered}
$$

The study was conducted on patients admitted in Nemazee hospital for chemotherapy in 2013. Inclusion criteria were all women with pathologically proven breast cancer who were younger than 65 years old; had advanced breast cancer; were in Karnofsky performance status equal or more than 70; and had normal renal and hepatic and heart functions. All women were aged more than 75 and had hypersensitivity to the chemotherapeutic agents; patients with distant metastasis and node-negative cases were excluded. According to the random numbers obtained from the site www.random.org, the patients were divided into two groups. Thirty-two women received chemotherapy regimen as: doxorubicin $60 \mathrm{mg} / \mathrm{m}^{2}$, cyclophosphamide $600 \mathrm{mg} / \mathrm{m}^{2}$ every 3 weeks for 4 cycles and thirty two women received 1chemotherapy regimen as: gemcitabine $1000 \mathrm{mg} / \mathrm{m}^{2}$, paclitaxel $175 \mathrm{mg} / \mathrm{m}^{2}$ every 3 weeks for 4 cycles. All the drugs were injected on the first day and Gemcitabine was injected on days 1 and 8 .

To determine the cost-utility of AC versus PG, the decision tree was used. These patients responded to chemotherapy in two forms: response, non-response. First, using this model, we calculated the expected costs and QALY for both methods and the incremental costeffectiveness ratio was measured; it was defined as the ratio of the difference between the expected cost and expected QALY. The decision tree is shown in Figure 1.

Data for this study was divided into two parts, utility and costs. The costs were identified and measured from community perspective. These items include medical and non- medical direct costs and indirect costs. In this study, a data collection form was used to collect the data; it consisted of two parts. The first part included demographic data of the patients and the second section contained information about the therapy and pharmaceutical costs, diagnosis and laboratory, the cost of accommodation and traveling and related expenses during chemotherapy. It should be noted that indirect costs are calculated using the Human Capital Approach and because the study period was one year, we did not use the discount rate. To assess

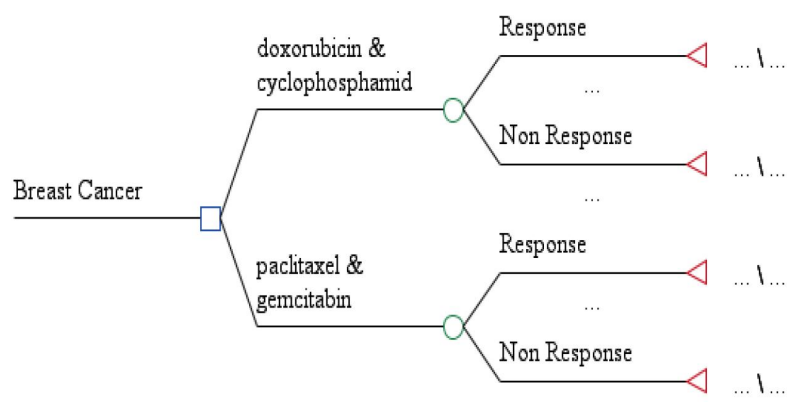

Figure 1. The Structure of Decision Tree 
Cost-Utility of "Doxorubicin and Cyclophosphamide" versus "Gemcitabine and Paclitaxel" for Breast Cancer in Iran

the utility of two regimens, the European Organization for Research and Treatment of Cancer Quality of Life Questionnaire-Core30 (EORTC QLQ-C30) was used. EORTC QLQ-C30 is a standardized questionnaire and its reliability and validity in the native language of 12 ethnic-cultural groups have been confirmed in Spain and the USA. Also in Iran, it was considered as an appropriate tool for measuring the quality of life in cancer patients with high reliability and validity, as mentioned by Montazeri et al (Montazeri et al., 1999).

This questionnaire consisted of performance scale (physical, role, emotional, cognitive and social), symptom scale (dyspnea, insomnia, appetite loss, constipation, diarrhea and financial difficulties, fatigue, nausea and vomiting, pain), and an overall health-status scale. In the questionnaire, the highest score in performance scale represents a better level of functioning and the highest score in overall health-status scale represents a better level of quality of life, but in symptom scales, high scores represent a worse level of symptoms. In general, this questionnaire reports separate scores for each dimension. First, using the weighted scores of quality of life, we calculated the utility (between zero and one). Finally, the QALY was measured by multiplying the amount of utility in the treatment period ( 3 months or 1.4 a year).

The data was collected in this part of the study by interviewing the patient after 3 months of follow-up, at the last session of chemotherapy for each participant. At the beginning of each interview, the overall goal of the project and interview was explained to each patient separately. Next, the researcher collected cost data through data collection forms and quality data by using a questionnaire (EORTC QLQ - C30). In this section, cost data and quality data were simultaneously obtained from the patient. However, during the interview, the interviewer was unaware of the treatment protocol because the study was designed as a double blind study. This increases the accuracy of the interview. To perform this analysis, Treeage 2011 and SPSS 16.0 specific softwares were used and descriptive statistical analysis was employed. Also, the Mann-Whitney test was used to determine the significant differences in costs between the two groups. By the decision tree, the expected costs and QALY were calculated and to increase the accuracy of the study, oneway deterministic sensitivity analysis (Tornado Diagram) and probabilistic sensitivity analyses were performed.

\section{Results}

Based on the results of the present study, among the 64 female patients studied, 68\% were married, 58\% were aged above 40 years, $12.5 \%$ were college educated, and $97 \%$ had insurance. The results are shown in Table 1. EORTCQLQ-C30 questionnaire has three scales: functional, symptoms and global health scales and the results are used to calculate QALY.

Analysis of the EORTC-QLQ-C30 questionnaire in the symptom scales showed that the average score for the role and social functioning in AC arm was significantly higher than PG arm. But the mean score of emotional functioning in $\mathrm{PG}$ arm was higher than $\mathrm{AC}$ arm. The physical performance and cognitive function in both arms were not statistically different.

The results of symptoms scale of the EORTCQLQ-C30 questionnaire showed that fatigue, pain, constipation and economic status in AC arm were significantly better (lower mean scores) compared to PG arm. While the nausea and vomiting, dyspnea, insomnia and diarrhea in the PG arm were better (lower mean scores) compared to PG arm. Comparison of loss of appetite in both groups showed no significant difference. Also, analysis of global health status of the questionnaire EORTC-QLQ-C30 in the last session of chemotherapy in both arms showed no significant difference. According to the results, in the AC arm, the QALY's mean was 0.1378 and in the PG arm, the QALY's mean was 0.1247.

According to the Table 3 , in both AC and PG arms, the mean of medical direct costs was the highest (1429.1 and 1944.3 US\$, respectively) and that of indirect costs was minimum (57.97 and 57.59 US\$, respectively). As seen in Table 4 , the mean of medical direct costs was significant

Table 1. Frequency Based on the Demographic Characteristics of Breast Cancer Patients

\begin{tabular}{llcc}
\hline Variable & & Number & Percent \\
\hline Age (year) & $<40$ & 27 & 42 \\
& $\geq 40$ & 37 & 58 \\
Marital Status & Married & 44 & 68 \\
& Single & 20 & 32 \\
Education & Collegiate Education & 8 & 12.5 \\
& Non-Collegiate Education & 56 & 87.5 \\
Insurance & Yes & 62 & 97 \\
& No & 2 & 3 \\
\hline
\end{tabular}

Table 2. QOL in AC and PG at the Last Session of chemotherapy

\begin{tabular}{lccl}
\hline Items of QoL & \multicolumn{2}{c}{ Chemotherapy regimen } & P value \\
Questionnaire & AC & PG & \\
\hline Functional scales* & & & \\
Physical functioning & $74.57 \pm 19.11$ & $67.07 \pm 22.33$ & 0.12 \\
Role functioning & $65.62 \pm 16.89$ & $52.59 \pm 22.04$ & 0.01 \\
Emotional functioning & $55.46 \pm 13.81$ & $65.62 \pm 17.80$ & 0.02 \\
Cognitive functioning & $67.18 \pm 16.11$ & $60.93 \pm 21.83$ & 0.46 \\
Social functioning & $70.82 \pm 14.66$ & $57.28 \pm 19.37$ & 0.005 \\
Symptom scales** & & & \\
Fatigue & $36.80 \pm 15.82$ & $50.34 \pm 20.92$ & 0.008 \\
Nausea and vomiting & $34.37 \pm 24.29$ & $21.87 \pm 19.60$ & 0.03 \\
Pain & $29.16 \pm 23.18$ & $41.66 \pm 26.09$ & 0.08 \\
Dyspnea & $21.8 \pm 27.5$ & $5.2 \pm 14.9$ & 0.005 \\
Insomnia & $45.8 \pm 33.6$ & $27.08 \pm 26$ & 0.02 \\
Appetite loss & $38.53 \pm 28.21$ & $39.57 \pm 27.35$ & 0.7 \\
Constipation & $23.95 \pm 30.79$ & $46.97 \pm 30.20$ & 0.002 \\
Diarrhea & $8.33 \pm 14.66$ & $2.08 \pm 8.19$ & 0.04 \\
Financial difficulties & & $69.78 \pm 25.9$ & 0.02 \\
Global health status* & $53.64 \pm 19.73$ & $47.11 \pm 17.63$ & 0.16 \\
\hline
\end{tabular}

Table 3. The Cost of Breast Cancer Patients Based on the Type of Costs (US\$)

\begin{tabular}{lrrr}
\hline Costs mean & \multicolumn{2}{c}{ Chemotherapy regimen } & P value \\
& AC & PG & \\
\hline Medical direct costs & $1429.1 \pm 506.07$ & $1944.3 \pm 658.94$ & 0.0001 \\
Non-medical direct costs & $66.86 \pm 32.21$ & $75.46 \pm 46.68$ & 0.545 \\
Indirect costs & $57.97 \pm 38.77$ & $57.59 \pm 55.98$ & 0.249 \\
Total & $1683.57 \pm 301.03$ & $2208.06 \pm 421.19$ & 0.123 \\
\hline
\end{tabular}




\section{Nahid Hatam et al}

in both $\mathrm{AC}$ and PG arms (p-value=0.003). But the means of non-medical direct costs and indirect costs were not significant different.

As seen in Table 4, the mean cost of chemotherapy was the highest medical direct costs in both arms; in the AC arm it was 1157.39 US\$ and in the PG arm 1646.39 US\$. Also, travel costs with 23.40 US\$ and auxiliary equipment costs with 28.19 US\$ were respectively the highest type of non-medical direct costs in AC and PG arms. The patients' accompany costs were the highest type of indirect costs in both arms. (In the AC arm 33.43 US\$ and in the PG arm 34.75 US\$, respectively)

The results of decision tree showed that in the AC arm, the expected cost was 39170.53 US\$ and the expected QALY was 3.39; also, in the PG arm, the expected cost was 43336.69 US\$ and the expected QALY was 2.64. Thus, as shown in the Figure, AC was dominant as compared to PG. According to Figure 2, AC is more cost-effective, as compared to $\mathrm{PG}$, because $\mathrm{AC}$ has more QALY and

Table 4. The Cost Components of AC and PG Regimen in Breast Cancer as Included in the Analysis (US\$)

\begin{tabular}{lcc}
\hline Type of cost & Chemotherapy regimen \\
& AC & PG \\
\hline Medical direct costs & & \\
Chemotherapy & $494.59 \pm 1157.39$ & $638.69 \pm 1646.39$ \\
Visits & $17.18 \pm 28.53$ & $16.29 \pm 28.89$ \\
Laboratory & $49.65 \pm 81.18$ & $60.58 \pm 96.17$ \\
Radiology & $89.27 \pm 162.05$ & $84.15 \pm 172.84$ \\
Non-medical direct costs & & \\
Traveling & $25.88 \pm 23.40$ & $21.61 \pm 23.90$ \\
Lodging and food & $10.90 \pm 11.48$ & $15.31 \pm 12.42$ \\
Phone & $4.57 \pm 9.84$ & $7.48 \pm 10.05$ \\
Auxiliary Equipment & $25.86 \pm 22.14$ & $29.48 \pm 28.19$ \\
Indirect costs & & \\
Time spent by the patient & $29.59 \pm 22.51$ & $31.39 \pm 22.83$ \\
Time spent by the patient's accompany & \\
& $31.10 \pm 33.43$ & $44.66 \pm 34.75$ \\
\hline
\end{tabular}

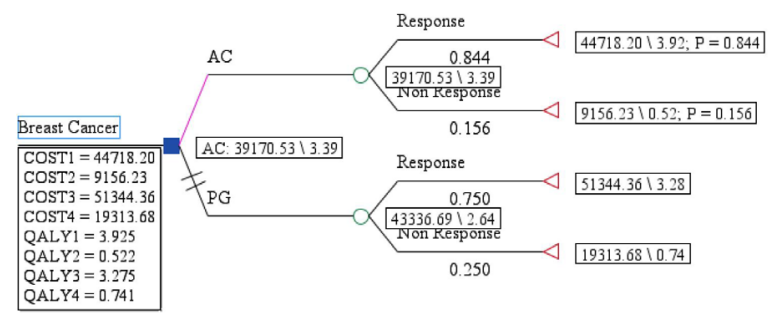

Figure 2. Results of the Decision Tree AC Versus PG

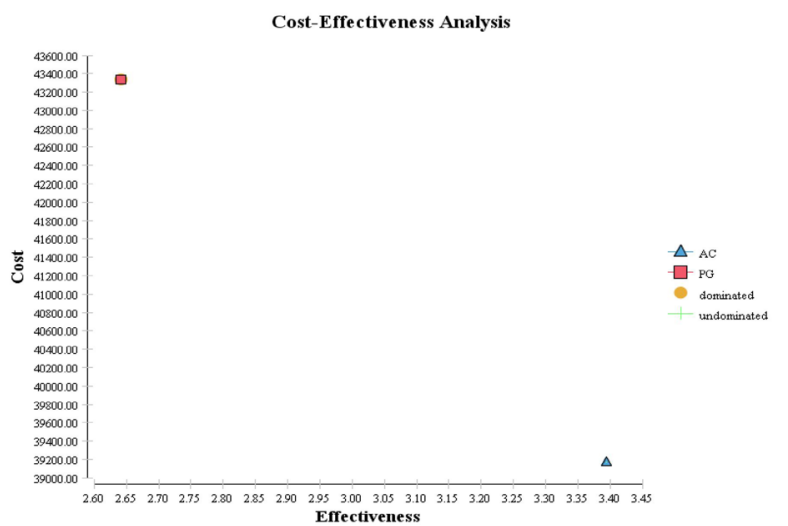

Figure 3. Cost-effectiveness Analysis of AC Versus PG

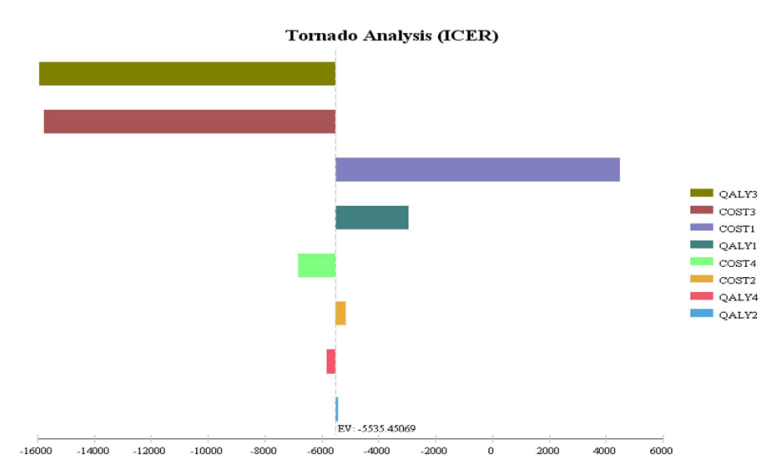

Figure 4. Results of One-Way Sensitivity Analysis (Tornado Diagram)

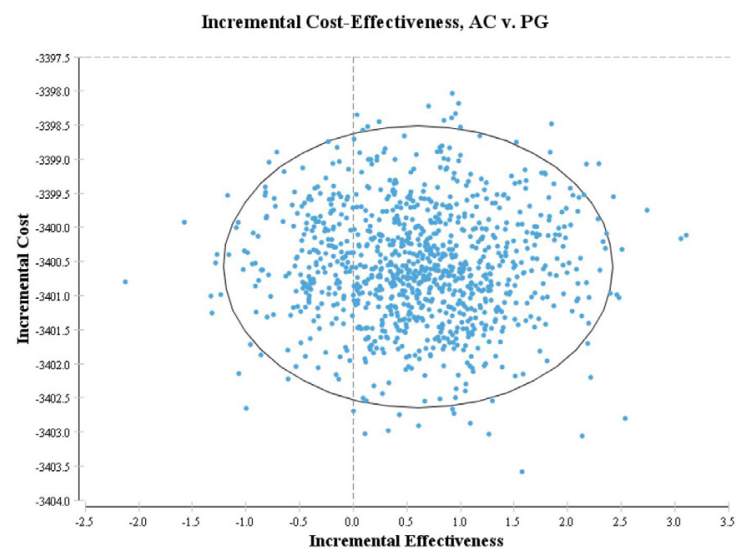

Figure 5. Incremental Cost-effectiveness Plane

less cost, as shown with triangle in Figure. But PG that is shown with the square has less QALY and more cost and is marked with a circle as the dominant option.

\section{Sensitivity nalysis}

Sensitivity Analysis provides a way that you can focus objectively on what is important (38). In this study, one-way sensitive analysis and probabilistic sensitivity analysis were done.

In Figure 3, we increased all parameters to $20 \%$. We also investigated how changes in model parameters would affect the total case averted using one-way sensi-tivity. Our results showed that changes in most of the input parameters had a few effects on the outcome. The total case averted were, especially, high sensitivity to QALY of patients who respond to PG and low sensitivity to the QALY of patients who did not respond to AC.

The incremental cost-effectiveness plane shows Monte Carlo estimates of incremental costs and QALY of using AC for treatment of breast cancer versus PG. For each one of the 10,000 iterations, values for parameters were randomly selected from their distributions and an ICER was calculated. AC was found to be a dominant strategy (less costly, more effective) in $97 \%$ of the simulations.

\section{Discussion}

The purpose of each type of economic evaluation, particularly in the field of medical decisions, is to improve the awareness of policy makers about the costs and effectiveness of the rival pharmaceutical and therapeutic technology and interventions (Akobundu et al., 2006). 
Cost-Utility of "Doxorubicin and Cyclophosphamide" versus "Gemcitabine and Paclitaxel" for Breast Cancer in Iran

The purpose of this study was to evaluate the cost-utility of chemotherapy regimens containing doxorubicin and cyclophosphamide compared to Gemcitabine and Paclitaxel in patients with advanced breast cancer. The findings of this study showed that in both AC and PG arms, the mean of medical direct costs was the highest (1429.1 and 1944.3 US\$, respectively) and the difference was significant ( $\mathrm{p}$-value $=0.003)$. One of the main reasons for higher costs of PG arm compared to AC arm was the cost of chemotherapy drugs. But the means of non-medical direct costs and indirect costs were not significantly different $(p$ value $=0.722$, $p$ value $=0.527)$. Also, the mean cost of chemotherapy was the highest medical direct costs in both arms; in the AC arm it was 1157.39 US\$ and in the PG arm it was 1646.39 US\$.

In the study of Haghighat et al. in 2013, the highest costs in stages II and III of breast cancer (56\% and 57\%, respectively) were related to chemotherapy (Haghighat et al., 2013). In the study by Nguyen Hoang Lan in Vietnam it was estimated that $64.9 \%$ of total health care costs in breast cancer is related to chemotherapy (Lan et al., 2013).

The findings of this study showed that chemotherapy costs was $74 \%$ of the total costs in the AC arm and $79 \%$ of those in PG arm. Direct costs measure the opportunity cost of the used resources in treatment of a specific disease while indirect costs measures the value of the lost resources in a particular disease. The findings of the study in America that examined the cost of metastatic breast cancer showed that the cost of treatment was $44 \%$, the cost of palliative and supportive was $31 \%$ and lost productivity was $21 \%$ of the total cost of metastatic breast cancer (Sorensen et al., 2012). In the study of Bernard, $70 \%$ of the total costs in AC arm was related to the cost of chemotherapy (Bernard et al., 2011).

According to the findings of this study, the average cost of chemotherapy regimens PG with 2208 US\$ was more than that of AC chemotherapy regimens with 1683.57 US\$ and the difference was significant ( $\mathrm{p}$ value $=0.001$ ). One of the main reasons for higher costs of $\mathrm{PG}$ arm compared to AC arm was the cost of chemotherapy drugs.

Chen and colleagues in their study in 2001 concluded that the costs of chemotherapy regimens containing Paclitaxel \& Gemcitabin were more than those of chemotherapy regimens containing Paclitaxel \& Carboplatin. They showed that the high cost of the PG regimen was due to the costs of chemotherapy drugs in PG regimen and the Gemcitabin on the eighth day (Chen et al., 2002).

Bernard et al in 2011, Younis et al in 2011 and Liubao in separate studies showed that the low cost of the AC regimen was due to the costs of chemotherapy drugs in AC regimen (Liubao et al., 2009; Bernard et al., 2011; Younis et al., 2011).

QALY calculation using the EORTC QLQ-C30 quality of life questionnaire in this study showed that in the AC arm, the QALY's mean was 0.1378 and in the PG arm, the it was 0.1247 ; the difference was not significant $(\mathrm{P}$ Value $=0.2$ )

The quality of life has been suggested as a part of the assessment criteria for treatment of cancer. In recent years, a comprehensive evaluation of cancer patients has had a special significance and Been exceeded the traditional examination of the biomedical implications and it has engulfed the effects of the disease on quality of life.

Ruston et al. showed that Cancer can cause various complications and has different effects on quality of life, Also, studies have shown that different chemotherapy treatments with different doses have different effects on the patients' utility (Rustoen, 1995).

One of the most common treatments of cancer is chemotherapy that causes long-term improvement in these patients. Despite the systematic destruction of the cancer cell, it also affects the healthy cells. The toxicity of chemotherapy drugs causes side effects such as bone marrow suppression and immune system, liver toxicity, skin disorders, central nervous system disorders, genitourinary and gastrointestinal complications, such as inflammation of the lining of the mouth and intestines (Del Gaudio and Menonna-Quinn, 1998; Omidvari et al., 2015).

Results of Hurny's study showed that there was a significant relationship between the chemotherapy and the quality of life of women with breast cancer (Hurny et al., 1996).

Stein and colleagues showed in their study that women with breast cancer undergoing radiotherapy and chemotherapy suffer poor sleep quality and thus have a lower quality of life (Stein et al., 2000).The results of the analysis of the EORTC-QLQ-C30 questionnaire at the last session of chemotherapy in this study showed that the greatest problem in the functional scales for patients receiving $\mathrm{AC}$ chemotherapy regimen was related to the emotional function and for patients receiving PG chemotherapy the regimen was related to the role function.

Analysis of the EORTC-QLQ-C30 questionnaire in the symptom scales showed that financial difficulties has received the highest rating in both arms and higher points in this scale represents the worse situation.

Fatigue has a profound negative impact on the quality of life in patients with cancer, and it creates a great interaction with the function patient. Fatigue is a common symptom experienced by cancer patients (Adamsen et al., 2004) and has been reported with a prevalence of 60$100 \%$, considering the cancer type, stage and prescribed treatment (Ream et al., 2002).

In this study, the most common complication caused by treatment in patients receiving PG chemotherapy regimen was fatigue. The mean score of fatigue in patients receiving $\mathrm{PG}$ chemotherapy regimen was significantly more than patients receiving $\mathrm{AC}$ chemotherapy regimen and higher average scores in this scale represent the worse situation.

The findings also showed that, in addition to fatigue, pain, constipation and economic status in the AC arm were significantly in a better situation (lower mean scores) compared to PG arm.

While the symptoms of nausea and vomiting, dyspnoea, insomnia and diarrhea in PG arm compared to $\mathrm{AC}$ has are in a better situation (lower mean scores) while that comparison of loss of appetite in both groups showed no significant difference. Analysis of the Global health status in the last session of chemotherapy in the two groups showed no significant difference between AC and PG. 
Based on the results of the study, AC is cost-effective, as compared to PG, and it is dominated because the expected cost was 39170.53 US\$ and the expected QALY was 3.39 in the AC arm whereas the expected cost was 43336.69 US\$ and the expected QALY was 2.64 in the PG arm. Therefore, AC is dominant compared to PG (less costly, more effective). It can be due to the higher cost of chemotherapy drugs in the PG and probably the Gemcitabin on the eighth day. Based on the results of the sensitivity analysis, ICER was highly sensitive to QALY of patients who responded to PG and was less sensitive to the QALY of patients who did not respond to AC.

In conclusion, Based on the results of the study, AC is cost-effective, as compared to PG, and it is dominated because the expected cost was 39170.53 dollars and the expected QALY was 3.39 in the AC arm whereas the expected cost was 43336.69 dollars and the expected QALY was 2.64 in the PG arm. Also, ICER was -5535.45 dollars (using AC saves 5535.45 dollars per each additional QALY). Therefore, it is recommended that oncologists should use AC instead of PG in the treatment of these patients.

\section{Acknowledgements}

This research was performed by Mr. Javad JavanNoghabi, in partial fulfillment of the requirements for certification as an MSc in Health Economic School at Shiraz University of Medical Sciences in Shiraz, Iran. The present article was adopted from the proposal number 937119 approved by Vice-Chancellor for Research Affairs of Shiraz University of Medical Sciences. The authors would like to thank Dr. Nasrin Shokrpour at Center for Development of Clinical Research of Nemazee Hospital for editorial assistance.

\section{References}

Adamsen L, Midtgaard J, Andersen C, et al (2004). Transforming the nature of fatigue through exercise: qualitative findings from a multidimensional exercise programme in cancer patients undergoing chemotherapy. Eur J Cancer Care (Engl), 13, 362-70.

Akobundu E, Ju J, Blatt L, et al (2006). Cost-of-illness studies : a review of current methods. Pharmacoeconomics, 24, 869-90.

Armour EP, White JR, DeWitt CC, et al (1996). Effects of continuous low-dose-rate brachytherapy on the rectum of the rat. Radiat Res, 145, 474-80.

Bernard LM, Verma S, Thompson MF, et al (2011). A Canadian economic analysis of U.S. Oncology Adjuvant Trial 9735. Curr Oncol, 18, 67-75.

Chen YM, Perng RP, Lee YC, et al (2002). Paclitaxel plus carboplatin, compared with paclitaxel plus gemcitabine, shows similar efficacy while more cost-effective: a randomized phase II study of combination chemotherapy against inoperable non-small-cell lung cancer previously untreated. Ann Oncol, 13, 108-15.

Davari M, Yazdanpanah F, Aslani A, et al (2013). The Direct Medical Costs of Breast Cancer in Iran: Analyzing the Patient's Level Data from a Cancer Specific Hospital in Isfahan. Int $J$ Prev Med, 4, 748-54.

Del Gaudio D, Menonna-Quinn D (1998). Chemotherapy. Potential occupational hazards. Am J Nurs, 98, 59-65.

Ferlay J, Autier P, Boniol M, et al (2007). Estimates of the cancer incidence and mortality in Europe in 2006. Ann Oncol, 18, 581-92.

Groot MT, Baltussen R, Uyl-de Groot CA, et al (2006). Costs and health effects of breast cancer interventions in epidemiologically different regions of Africa, North America, and Asia. Breast J, 12, 81-90.

Haghighat s, akbari e, ghafari s, et al (2013). economic burden of Diagnostic and therapeutic implications in nonmetastatic breast cancer. Journal of Breast Disease, 6.

Hornberger JC, Best JH (2005). Cost utility in the United States of rituximab plus cyclophosphamide, doxorubicin, vincristine, and prednisone for the treatment of elderly patients with diffuse large B-cell lymphoma. Cancer, 103, 1644-51.

Hurny C, Bernhard J, Coates AS, et al (1996). Impact of adjuvant therapy on quality of life in women with node-positive operable breast cancer. International Breast Cancer Study Group. Lancet, 347, 1279-84.

Jemal A, Bray F, Center MM, et al (2011). Global cancer statistics. CA Cancer J Clin, 61, 69-90.

Kolahdoozan S, Sadjadi A, Radmard AR, et al (2010). Five common cancers in Iran. Arch Iran Med, 13, 143-6.

Lan NH, Laohasiriwong W, Stewart JF, et al (2013). Cost of treatment for breast cancer in central Vietnam. Global health action, $\mathbf{6}$.

Liubao P, Xiaomin W, Chongqing T, et al (2009). Cost-effectiveness analysis of adjuvant therapy for operable breast cancer from a Chinese perspective: doxorubicin plus cyclophosphamide versus docetaxel plus cyclophosphamide. Pharmacoeconomics, 27, 873-86.

Marsland T, Robbins G, Marks A, et al (2010). Reducing cancer costs and improving quality through collaboration with payers: a proposal from the Florida society of clinical oncology. $J$ Oncol Pract, 6, 265-9.

Mohammadianpanah M, Ashouri Y, Hoseini S, et al (2012). The efficacy and safety of neoadjuvant chemotherapy $+/$ - letrozole in postmenopausal women with locally advanced breast cancer: a randomized phase III clinical trial. Breast Cancer Res Treat, 132, 853-61.

Montazeri A, Harirchi I, Vahdani M, et al (1999). The European Organization for Research and Treatment of Cancer Quality of Life Questionnaire (EORTC QLQ-C30): translation and validation study of the Iranian version. Support Care Cancer, 7, 400-6.

Mousavi SM, Gouya MM, Ramazani R, et al (2009). Cancer incidence and mortality in Iran. Ann Oncol, 20, 556-63.

Omidvari S, Razzaghi S, Zamani A, et al (2015). Rate and Time of Ovarian Function Restoration in Menopausal Breast Cancer Patients Who Received Letrozole Following Chemotherapy. Middle East Journal of Cancer, 6, 35-42.

Papadimitriou K, Ardavanis A, Kountourakis P (2010). Neoadjuvant therapy for locally advanced breast cancer: Focus on chemotherapy and biological targeted treatments' armamentarium. J Thorac Dis, 2, 160-70.

Ream E, Richardson A, Alexander-Dann C (2002). Facilitating patients' coping with fatigue during chemotherapy-pilot outcomes. Cancer Nurs, 25, 300-8.

Rustoen T (1995). Hope and quality of life, two central issues for cancer patients: a theoretical analysis. Cancer Nurs, 18, 355-61.

Shih YC, Halpern MT (2008). Economic evaluations of medical care interventions for cancer patients: how, why, and what does it mean? CA Cancer J Clin, 58, 231-44.

Sorensen SV, Goh JW, Pan F, et al (2012). Incidence-based cost-ofillness model for metastatic breast cancer in the United States. Int J Technol Assess Health Care, 28, 12-21.

Stein KD, Jacobsen PB, Hann DM, et al (2000). Impact of hot flashes on quality of life among postmenopausal women being treated for breast cancer. J Pain Symptom Manage, 19, 436-45.

Younis T, Rayson D, Skedgel C (2011). The cost-utility of adjuvant chemotherapy using docetaxel and cyclophosphamide compared with doxorubicin and cyclophosphamide in breast cancer. Curr Oncol, 18, 288-96. 